\title{
Genetic Abnormalities in Biliary Brush Samples for Distinguishing Cholangiocarcinoma from Benign Strictures in Primary Sclerosing Cholangitis
}

\author{
Margriet R. Timmer, ${ }^{1,2}$ Chiu T. Lau, ${ }^{1,2}$ Sybren L. Meijer, ${ }^{3}$ Paul Fockens, ${ }^{1}$ Erik A. J. Rauws, \\ Cyriel Y. Ponsioen, ${ }^{1}$ Silvia Calpe, ${ }^{1,2}$ and Kausilia K. Krishnadath ${ }^{1,2}$ \\ ${ }^{1}$ Department of Gastroenterology and Hepatology, Academic Medical Center, 1100 DD Amsterdam, Netherlands \\ ${ }^{2}$ Center for Experimental and Molecular Medicine, Academic Medical Center, 1100 DD Amsterdam, Netherlands \\ ${ }^{3}$ Department of Pathology, Academic Medical Center, 1100 DD Amsterdam, Netherlands
}

Correspondence should be addressed to Kausilia K. Krishnadath; k.k.krishnadath@amc.uva.nl

Received 21 December 2015; Accepted 22 February 2016

Academic Editor: Keiichi K. Kubota

Copyright (C) 2016 Margriet R. Timmer et al. This is an open access article distributed under the Creative Commons Attribution License, which permits unrestricted use, distribution, and reproduction in any medium, provided the original work is properly cited.

\begin{abstract}
Background. Primary sclerosing cholangitis (PSC) is a chronic inflammatory liver disease and is strongly associated with cholangiocarcinoma (CCA). The lack of efficient diagnostic methods for CCA is a major problem. Testing for genetic abnormalities may increase the diagnostic value of cytology. Methods. We assessed genetic abnormalities for CDKN2A, TP53, ERBB2, 20q, MYC, and chromosomes 7 and 17 and measures of genetic clonal diversity in brush samples from 29 PSC patients with benign biliary strictures and 12 patients with sporadic CCA or PSC-associated CCA. Diagnostic performance of cytology alone and in combination with genetic markers was evaluated by sensitivity, specificity, and area under the curve analysis. Results. The presence of MYC gain and CDKN2A loss as well as a higher clonal diversity was significantly associated with malignancy. MYC gain increased the sensitivity of cytology from $50 \%$ to $83 \%$. However, the specificity decreased from $97 \%$ to $76 \%$. The diagnostic accuracy of the best performing measures of clonal diversity was similar to the combination of cytology and MYC. Adding CDKN2A loss to the panel had no additional benefit. Conclusion. Evaluation of MYC abnormalities and measures of clonal diversity in brush cytology specimens may be of clinical value in distinguishing CCA from benign biliary strictures in PSC.
\end{abstract}

\section{Introduction}

Primary sclerosing cholangitis (PSC) is a cholestatic liver disease characterized by diffuse inflammation and progressive stricturing of the intra- and extrahepatic bile ducts, which may eventually lead to liver cirrhosis. Patients with PSC have an increased risk of developing cholangiocarcinoma (CCA) with a lifetime incidence of approximately $10-20 \%$. Accurate detection of CCA is of paramount importance to select patients for curative treatment options such as surgical resection or liver transplantation $[1,2]$. Therefore, patients presenting with a dominant biliary stricture should be thoroughly evaluated for the presence of a CCA [3]. The diagnostic approach includes imaging modalities, serum levels of the tumor marker Ca19-9 as well as biopsies, and brush cytology of the bile ducts obtained during endoscopic retrograde cholangiopancreatography (ERCP). However, benign inflammatory strictures that are part of the natural history of PSC are often difficult to distinguish from malignant strictures. Imaging techniques and Ca19-9 have limited sensitivity, while increased Ca19-9 levels can also be found in PSC in the absence of malignancy [4-6]. Conventional cytology is highly specific (95\%-100\%) for diagnosing CCA, but its sensitivity is generally low and varies between $12 \%$ and $62 \%$ [7-10]. The use of molecular markers could improve the diagnostic value of cytology.

Genetic abnormalities that are involved in the process of malignant progression provide a source of potential biomarkers $[5,11-14]$. In PSC, both the release of inflammatory cytokines and the toxic effect of bile acids during cholestasis 
seem to contribute to the occurrence of genetic abnormalities and malignant transformation of cholangiocytes [1517]. The diagnostic value of DNA content abnormalities in brushing specimens has been evaluated in several studies using different techniques including fluorescence in situ hybridization (FISH) and flow cytometry and has been shown to have a sensitivity of around $50 \%[5,18]$. The use of DNA content abnormalities is mainly based on the assessment of chromosome copy number alterations as a diagnostic feature of malignancy, whereas reports on the assessment of losses or gains of particular tumor suppressor genes or oncogenes are scarce $[19,20]$.

Measurement of clonal diversity is a novel method that can be used to risk stratify precancerous lesions. It is assumed that cancers evolve by a reiterative process of clonal expansion, genetic diversification, and clonal selection, which also depends on environmental conditions. Although cancer clonal dynamics can be complex and result in highly variable patterns of genetic diversity, innovative measures of clonal diversity have been shown to be of prognostic value in several malignant and premalignant conditions including Barrett's esophagus and esophageal adenocarcinoma [21]. As far as we know these measures have not been studied in PSC.

Because reliable markers for detection of CCA are currently not available, we aimed to evaluate a novel panel of DNA FISH markers and markers of clonal diversity in cytology specimens of patients with PSC, PSC-associated CCA, and CCA to further clarify the process of malignant degeneration and see if they could serve as tumor markers with the aim of improving clinical management. Our panel included the centromeric probes of chromosomes 7 and 17, locus-specific probes to the tumor suppressor genes CDKN2A (p16) and TP53, and three oncogenes including ERBB2 (Her2/neu), 20q, and MYC. In previous studies, all seven markers have been shown to be common alterations in bile duct malignancy, although the frequencies vary widely depending on the techniques used [17, 19, 22-27]. In addition, we tested if, based on this set of markers, measures of genetic clonal diversity have potential for diagnosing malignancy in biliary strictures.

\section{Materials and Methods}

2.1. Study Design and Patients. We prospectively included all consecutive patients with well-defined PSC with suspected biliary strictures who underwent ERCP in our tertiary referral center between January 2012 and April 2014. The diagnosis of PSC was based on the presence of characteristic bile duct changes including multifocal strictures and segmental dilatations on cholangiography (ERCP or magnetic resonance cholangiopancreatography) and a compatible cholestatic biochemical profile, after exclusion of other causes of secondary sclerosing cholangitis. In addition, brush cytology specimens were obtained from patients with sporadic CCA, while negative control specimens were obtained from the duodenum of patients who underwent upper endoscopy to evaluate upper gastrointestinal symptoms during which no endoscopic abnormalities were found. Demographic data and corresponding cytological results were recorded in a prospectively maintained database. The local ethics committee approved the study and all patients provided informed consent.

2.2. Specimen Collection and Conventional Cytology. Cytology samples were taken from the strictures using a cytology brush compiled of stiff and soft bristles (Infinity ${ }^{\mathrm{TM}}$, US Endoscopy, Mentor, $\mathrm{OH}$ ) by moving the brush several times back and forth through the stricture. The brush was cut into two equal parts and both were placed in a separate vial with $20 \mathrm{~mL}$ PreservCyt solution (Hologic, Marlborough, MA). The first vial was used for conventional cytology and analyzed per standard practice by a pathologist. Cytologic diagnoses were categorized as "negative," "atypical," "suspicious," or "malignant" using earlier published criteria [28, 29]. Representative examples of biliary brushing specimens are shown in Figures 1 (a) and 1(b).

2.3. Fluorescence In Situ Hybridization. Cells were released from the brush by rigorous shaking and concentrated in $3 \mathrm{~mL}$ by removal of the supernatant. The cytospin procedure (Shandon Cytospin 4, Cytocentrifuge, Thermoscientific, Waltham, MA) was performed to concentrate the cells on a slide in a uniform monolayer after which the slides were stored at $-80^{\circ} \mathrm{C}$ until further preparation for FISH. FISH was performed as previously described using seven different DNA probes including the centromeric probes CEP7 and CEP17 and the locus-specific probes to CDKN2A, TP53, ERBB2, 20q, and MYC (Abbott Molecular, Abbott Park, Illinois) [30]. Probes were organized in two probe sets with set 1 comprising CEP17, ERBB2, TP53, and CDKN2A and set 2 comprising CEP7, CEP17, 20q, and MYC. Slides were reviewed by an experienced technician who was blinded to the patient's clinical history and cytology results. Slides were analyzed by manual counting of the number of signals for each of the probes in 100 (minimum 75) consecutive interphase nuclei of biliary epithelial cells. Nuclei with two signals for a particular probe were considered as normal. Data were recorded as the percentage of cells showing genetic abnormalities for a particular marker. For control purposes (to determine background hybridization variation), the probes in this study were also applied to duodenum brushes of 10 healthy control individuals. Cut-off values were calculated for each probe separately as the mean percentage of cells in duodenum specimens showing abnormalities plus twice the standard deviation, rounded up to the next integer. All samples were scored as positive or negative for each marker based on the cut-off values for each individual probe. Cut-off values were 1\% (CDKN2A loss), 3\% (TP53 loss), 0\% (ERBB2), 1\% (20q), 3\% (MYC), 3\% (CEP7), and 4\% (CEP17). Representative examples of DNA FISH of biliary brushing specimens are shown in Figures 1(c) and 1(d).

2.4. Genetic Clonal Diversity. Measures of clonal diversity were computed to quantify the degree of genetic diversity within a sample. Clones were defined as the collection of cells characterized by the same genotype based on 


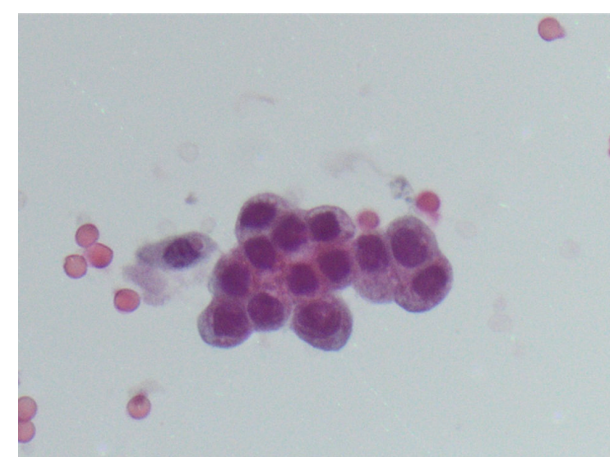

(a)

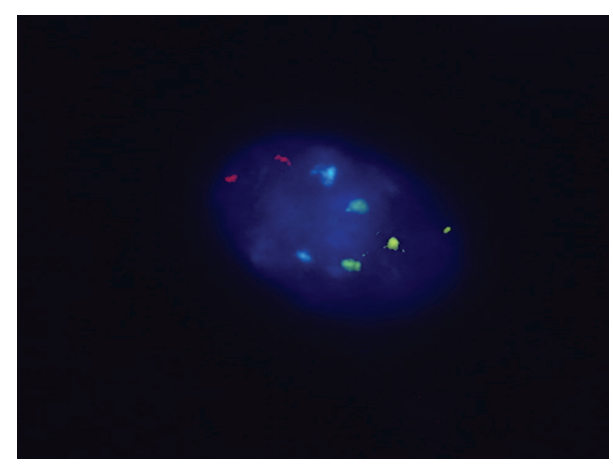

(c)

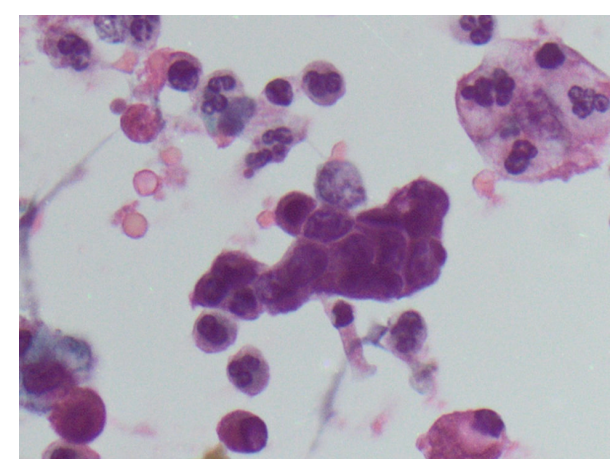

(b)

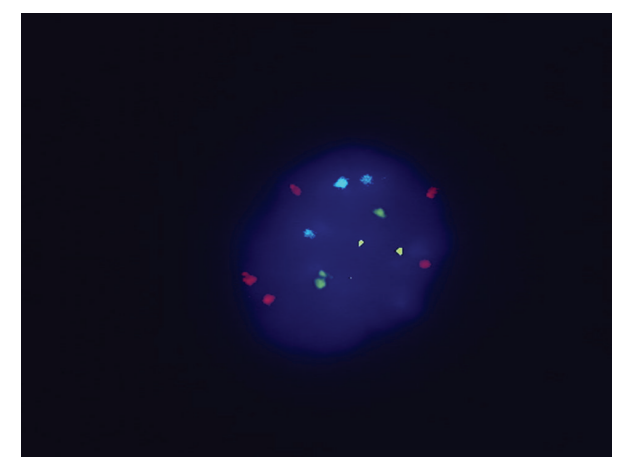

(d)

FIGURE 1: Representative examples of conventional cytology and DNA FISH of biliary brushing specimens. ((a) and (b)) Conventional cytology. (a) Reactive changes in the context of PSC with some architectural irregularity, mild variation in nuclear size, and overall intact nuclear to cytoplasmic ratio. (b) Adenocarcinoma in a patient with underlying PSC showing hyperchromatic nuclei, marked variation in nuclear shape and size with nuclear molding, and severely disturbed nuclear to cytoplasmic ratio. In the background, necrotic debris with degenerative cells and granulocytes are observed. ((c) and (d)) Representative examples of FISH signal patterns seen in biliary strictures with probes for CEP7 [aqua], CEP17 [green], 20q [gold], and MYC [red]. (c) A normal cell (2 signals of each probe) and (d) gain of $M Y C$ ( $>2$ red signals) and gain of CEP7 (>2 blue signals).

the combination of marker-specific copy numbers for the two probe sets used in this study. Thus, for each cell, the number of signals for each probe in a particular probe set was recorded and these data were used to identify different clones. We analyzed two indices of clonal diversity for both probe sets: richness and the Shannon index. Richness measures the number of different clones, while the Shannon index takes into account both the number of different clones and the abundance of clones [21]. The Shannon diversity index (S) is calculated as $S=\sum_{i=1}^{R} p_{i} \ln \left(p_{i}\right)$, where $R$ is the number of different clones in a sample and $p_{i}$ is the frequency of clone $i$ in the sample.

2.5. Gold Standard for Malignancy. Pathological diagnosis was considered the "gold standard" for diagnosing benign and malignant disease. Pathological evidence of carcinoma included positive routine cytology, histological confirmation of a CCA, or positive fine needle aspiration indicating metastatic disease. Strictures were classified as benign based on negative pathology results and a cancer-free clinical course of at least six months.
2.6. Statistical Analysis. All statistical analyses were performed using SPSS (version 21) and GraphPad Prism (version 5.01). Data were presented as mean \pm standard deviation (SD) or median and interquartile range (IQR) for quantitative variables and frequencies and percentages for categorical data. Groupwise comparisons of categorical data were analyzed by using Fisher's exact test and continuous measures were compared with the use of Student's $t$-test. Diagnostic performance of cytology alone and in combination with genetic markers was evaluated by sensitivity, specificity, and area under the curve (AUC) analysis. Receiver operator characteristic (ROC) curves were created to determine optimal cut-off values for different measures of diversity. Results were considered statistically significant at $P$ values $<0.05$.

\section{Results}

3.1. Patients. Brushes were obtained from 41 individual patients undergoing ERCP including 29 patients with PSC, 3 with PSC complicated by CCA, and 9 with CCA. In the majority of patients (97\%) a dominant stricture was found 
TABLE 1: Clinical characteristics and laboratory values of patient populations studied.

\begin{tabular}{lcc}
\hline & PSC & CCA \\
& $n=29$ & $n=12$ \\
\hline Male sex & $14(48 \%)$ & $7(58 \%)$ \\
Age, years & $43.5 \pm 13.0$ & $60.6 \pm 12.0$ \\
Jaundice & $11(38 \%)$ & $10(83)$ \\
Weight loss & $3(10 \%)$ & $7(58 \%)$ \\
Abdominal pain & $10(35 \%)$ & $2(17 \%)$ \\
Fatigue & $11(38 \%)$ & $5(42 \%)$ \\
IBD & $16(55 \%)$ & $2(17 \%)$ \\
AST, U/L & $86.9 \pm 69.7$ & $73.3 \pm 30.9$ \\
ALT, U/L & $102.0 \pm 88.4$ & $224.6 \pm 196.7$ \\
ALP, U/L & $365.7 \pm 215.5$ & $475.5 \pm 322.8$ \\
Bilirubin $\mu \mathrm{mol} / \mathrm{L}$ & $44.5 \pm 57.5$ & $157.3 \pm 184.0$ \\
\hline
\end{tabular}

Values are presented as number (\%) or mean \pm standard deviation. ALP: alkaline phosphatase; ALT: alanine aminotransferase; AST: aspartate aminotransferase; CCA: cholangiocarcinoma; IBD: inflammatory bowel disease; PSC: primary sclerosing cholangitis.

during ERCP. All patients had corresponding cytology and FISH results. Patients reported complaints of jaundice (51\%), fatigue (39\%), abdominal pain (29\%), and weight loss (24\%). Most patients $(81 \%)$ reported at least one of the abovementioned complaints. Patient characteristics and laboratory values at the time of ERCP are shown in Table 1. Within the group of PSC patients ( $n=32,17$ males, median age 47 years), malignancy was confirmed by conventional cytology $(n=$ $1)$, detection of metastatic disease by fine needle aspiration $(n=1)$, and, in one patient with a high suspicion of a CCA, the final diagnosis made at the time of surgical resection. In the remaining 29 PSC patients with a stricture classified as "benign," no malignancy was diagnosed during followup (median follow-up, 16 months, IQR, 8-27 months). The population of patients with a sporadic CCA consisted of 4 male and 5 female patients (mean age $63.4 \pm 12.5$ years).

3.2. Conventional Cytology. Overall, 26 samples (63\%) were classified as negative, $8(20 \%)$ were classified as atypical, four $(10 \%)$ were classified as suspicious, and three patients (7\%) had a brush that was positive for CCA. When only a "malignant" cytology result was considered positive for malignancy, this resulted in an overall sensitivity of $25 \%$ and a specificity of $100 \%$ (Table 2). The sensitivity and specificity were $50 \%$ and $97 \%$, respectively, when all specimens classified as "suspicious" or "malignant" were considered positive for malignancy. Among the PSC patients, cytology had a sensitivity of $33 \%$ and a specificity of $100 \%$. Of the 3 patients with a PSC-associated CCA, one had a "malignant" cytology result, one was reported as "atypical," and one brush was negative. In the PSC patients without malignancy, cytology from one patient was reported as "suspicious for malignancy." This patient underwent multiple ERCP during follow-up for short-term stenting and balloon dilatation of the stricture, but additional bile duct brushing (3 times) during a period of 19 months did not show any more signs of malignancy.
3.3. Genetic Abnormalities. First, we compared the presence of genetic abnormalities in malignant strictures (both PSC and non-PSC) to benign PSC strictures (Table 2). The markers $C D K N 2 A$ loss and $M Y C$ gain were significantly associated with malignancy and were observed in $67 \%$ and $58 \%$ of the CCAs and in $31 \%$ and $21 \%$ of the benign PSC strictures (Fisher's exact test, $P=0.045$ and $P=0.029$, resp.). Although gains of $C E P 7$ and $C E P 17$ were more frequently observed in CCA than in benign strictures (58\% versus $24 \%$ and $83 \%$ versus $55 \%$, resp.), these differences were not statistically significant $(P=0.068$ and $P=0.154)$. Similarly, there was no significant difference between gains of $20 q$ in malignant $(67 \%)$ and benign strictures $(41 \%)(P=0.18)$.

We evaluated the diagnostic performance of cytology and the best performing makers (i.e., the markers CDKN2A and $M Y C$ that were both significantly associated with CCA) in different combinations. The combination of cytology and FISH for MYC (considered positive when either cytology or FISH was positive) resulted in an increase in sensitivity from $50 \%$ to $83 \%$, but specificity decreased from $97 \%$ to $76 \%$. Combining cytology with FISH for both CDKN2A and MYC increased the sensitivity further to $92 \%$, but this was at the expense of lower specificity (52\%). This was also reflected by the AUC that was 0.73 for cytology alone, 0.80 for cytology combined with FISH analysis for MYC, and 0.72 for cytology combined with $C D K N 2 A$ and/or MYC. When we combined CDKN2A and MYC without cytology, 10 out of 12 CCA patients tested positive for at least one of the markers. However, of the 29 patients with benign strictures, also 13 patients had CDKN2A and/or MYC abnormalities. Sensitivity and specificity were $83 \%$ and $55 \%$, respectively.

When we restricted the analysis to PSC patients only, we observed that abnormalities of CDKN2A, 20q, CEP17, and $M Y C$ were more frequent in PSC-CCA than in CCA. However, only the difference in MYC abnormalities was a significant finding where $M Y C$ abnormalities were seen in $21 \%$ of benign PSC cases and in all cases of PSC-CCA ( $P=$ 0.017 ) (Figure 2). This corresponded to a sensitivity of $100 \%$ and a specificity of $79 \%$. Interestingly, MYC abnormalities were also seen more frequently in PSC-CCA than in sporadic CCA (100\% versus $44 \%)$. This difference was, however, not significant $(P=0.21)$.

3.4. Clonal Diversity. The number of different clones per sample (i.e., richness) was significantly higher in CCA compared with PSC. This was the case for both probe set 1 (mean 6.33 versus $4.14, P=0.007$ ) and probe set 2 (mean 6.50 versus $4.83, P=0.002$ ) (Figure 3 ). Also the Shannon diversity was significantly higher in CCA than in PSC when measured by probe set 1 (mean Shannon diversity of 0.51 versus $0.31, P=0.017$ ) and probe set 2 (mean 0.50 versus $0.36, P=0.002$ ). We computed ROC curves to compare the discriminative performance of the different measures of diversity. Richness (set 2) and Shannon diversity (set 1) had the highest discriminative value with both having an AUC of 0.78 (Table 2). Using a cut-off value of 5.5 for richness (set 2) and a cut-off value of 0.34 for Shannon diversity (set 1 ), this corresponded to a sensitivity of $83 \%$ and a specificity of $72 \%$. 
TABLE 2: Diagnostic performance of cytology, FISH, and measures of clonal diversity in distinguishing benign from malignant strictures.

\begin{tabular}{|c|c|c|c|c|c|c|}
\hline & $\begin{array}{c}\text { PSC } \\
n=29\end{array}$ & $\begin{array}{c}\text { CCA } \\
n=12\end{array}$ & $P$ value & Sensitivity & Specificity & AUC (95\% CI) \\
\hline \multicolumn{7}{|l|}{ Cytology } \\
\hline Cytology (M), n (\%) & $0(0)$ & $3(25)$ & 0.021 & $25 \%$ & $100 \%$ & $0.63(0.43-0.83)$ \\
\hline Cytology (S + M), $n(\%)$ & $1(3)$ & $6(50)$ & 0.001 & $50 \%$ & $97 \%$ & $0.73(0.54-0.93)$ \\
\hline \multicolumn{7}{|l|}{ FISH markers } \\
\hline CDKN2A loss, $n(\%)$ & $9(31)$ & $8(67)$ & 0.045 & $67 \%$ & $69 \%$ & $0.68(0.49-0.86)$ \\
\hline TP53 loss, $n(\%)$ & $1(3)$ & $2(17)$ & 0.20 & $17 \%$ & $97 \%$ & $0.57(0.36-0.77)$ \\
\hline$E R B B 2$ gain, $n(\%)$ & $2(7)$ & $0(0)$ & 1.00 & $0 \%$ & $93 \%$ & $0.47(0.28-0.66)$ \\
\hline $20 q$ gain, $n(\%)$ & $12(41)$ & $8(67)$ & 0.181 & $67 \%$ & $59 \%$ & $0.63(0.44-0.82)$ \\
\hline MYC gain, $n(\%)$ & $6(21)$ & $7(58)$ & 0.029 & $58 \%$ & $79 \%$ & $0.69(0.50-0.88)$ \\
\hline CEP7 gain, $n(\%)$ & $7(24)$ & $7(58)$ & 0.068 & $58 \%$ & $76 \%$ & $0.67(0.48-0.86)$ \\
\hline CEP17 gain, $n(\%)$ & $16(55)$ & $10(83)$ & 0.154 & $83 \%$ & $45 \%$ & $0.64(0.46-0.82)$ \\
\hline \multicolumn{7}{|l|}{ Cytology and FISH } \\
\hline Cytology $(\mathrm{S}+\mathrm{M})$ and $C D K N 2 A, n(\%)$ & $10(34)$ & $10(83)$ & 0.006 & $83 \%$ & $66 \%$ & $0.74(0.58-0.91)$ \\
\hline Cytology $(\mathrm{S}+\mathrm{M})$ and $M Y C, n(\%)$ & $7(24)$ & $10(83)$ & 0.001 & $83 \%$ & $76 \%$ & $0.80(0.64-0.95)$ \\
\hline Cytology $(\mathrm{S}+\mathrm{M})$ and $M Y C / C D K N 2 A, n(\%)$ & $14(48)$ & $11(92)$ & 0.013 & $92 \%$ & $52 \%$ & $0.72(0.56-0.88)$ \\
\hline \multicolumn{7}{|l|}{ Diversity measures } \\
\hline Richness (set 1) & 11 & 10 & 0.015 & $83 \%$ & $62 \%$ & $0.73(0.56-0.89)$ \\
\hline Richness (set 2) & 8 & 10 & 0.002 & $83 \%$ & $72 \%$ & $0.78(0.62-0.94)$ \\
\hline Shannon diversity (set 1 ) & 8 & 10 & 0.002 & $83 \%$ & $72 \%$ & $0.78(0.62-0.94)$ \\
\hline Shannon diversity (set 2) & 10 & 10 & 0.006 & $83 \%$ & $65 \%$ & $0.74(0.58-0.91)$ \\
\hline \multicolumn{7}{|l|}{ Diversity measures and cytology } \\
\hline Cytology and richness (set 2) & 9 & 11 & $<0.001$ & $92 \%$ & $69 \%$ & $0.80(0.66-0.95)$ \\
\hline Cytology and Shannon diversity (set 1) & 9 & 11 & $<0.001$ & $92 \%$ & $69 \%$ & $0.80(0.66-0.95)$ \\
\hline
\end{tabular}

$P$ values are compared using Fisher's exact test. M refers to malignant and S refers to suspicious. CCA includes 3 patients with PSC-associated CCA and 9 patients with sporadic CCA. AUC: area under the curve; CCA: cholangiocarcinoma; CI: confidence interval; PSC: primary sclerosing cholangitis; NA: not applicable. Cut-off values for measures of clonal diversity were determined using ROC curves and were 4.5 for richness (set 1), 5.5 for richness (set 2), 0.34 for Shannon diversity (set 1), and 0.43 for Shannon diversity (set 2).

Both measures increased the sensitivity of normal cytology from $50 \%$ to $92 \%$, although specificity went down to $69 \%$. Cytology and richness (set 2) combined had an AUC of 0.80 and cytology combined with Shannon diversity (set 1) also resulted in an AUC of 0.80 . However, the best performing diversity measures already had an AUC of 0.78 by itself.

\section{Discussion}

Brush cytology has limited sensitivity for diagnosing CCA as the cause of biliary strictures, which is important because accurate diagnosis of early-stage CCA could increase the number of patients suitable for surgical resection or liver transplantation. Surveillance is therefore predicated on the availability of reliable diagnostic tools. Because most CCAs develop in the perihilar region or in the extrahepatic bile ducts, brushing and biopsy specimens of the biliary tract still represent the most reliable method for early detection of malignant degeneration. CCAs are often surrounded by dense, desmoplastic stroma and in a significant number of cases cytology specimens do not contain sufficient epithelial cells for interpretation. The use of FISH enables the detection of genetic abnormalities, even when these abnormalities are only present in a small fraction of cells, and FISH is therefore an attractive diagnostic tool.

Previous studies have revealed several genetic abnormalities involved in malignant progression in CCA. However, studies often focused on single genetic events, while the assessment of genetic diversity may provide additional information about the genetic profile of the disease, which cannot be obtained from a single marker. This may ultimately lead to improved diagnostic and prognostic tools. Multicolor DNA FISH on cytology specimens as applied in this study is an excellent method for identifying subclones in cellular specimens, which can be used to determine diversity status. Therefore, we not only assessed conventional biomarker status of single events but also used the frequency and abundance of genetic alterations at multiple loci to measure indices of clonal diversity.

We included 29 patients with well-defined PSC and 12 patients with CCA. Patients were prospectively followed up during a median of 16 months to assure that all patients without CCA were truly "benign" patients. We observed that abnormalities of $M Y C$ and CDKN2A were significantly associated with the presence of malignancy. As expected, abnormalities of CEP7, CEP17, and $20 q$ were more frequently noticed in malignancy, but the differences between the 

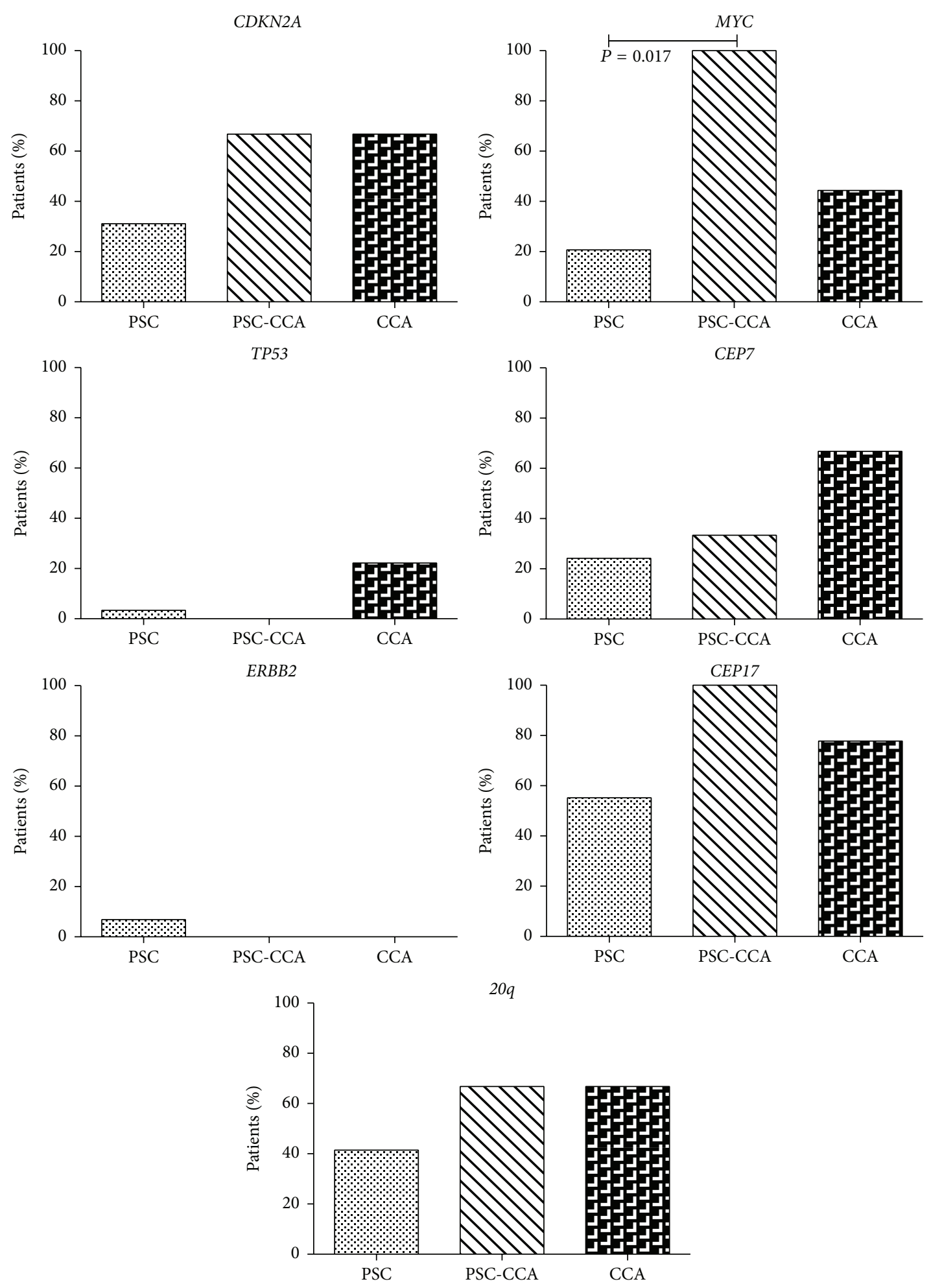

FIgURE 2: Genetic abnormalities in PSC, PSC-associated CCA, and sporadic CCA. $P$ values are compared using Fisher's exact test.

benign and malignant cases were not statistically significant in our dataset. Combining cytology with $M Y C$ aberrations increased the sensitivity from $50 \%$ to $85 \%$, however with a decrease in specificity from $97 \%$ to $76 \%$. As has been previously reported, $C D K N 2 A$ loss was also significantly associated with CCA but it did not improve the diagnostic performance beyond the use of MYC [19].

Furthermore, we demonstrated for the first time that levels of clonal diversity are significantly higher in CCA compared to benign PSC strictures. This is in line with previous 

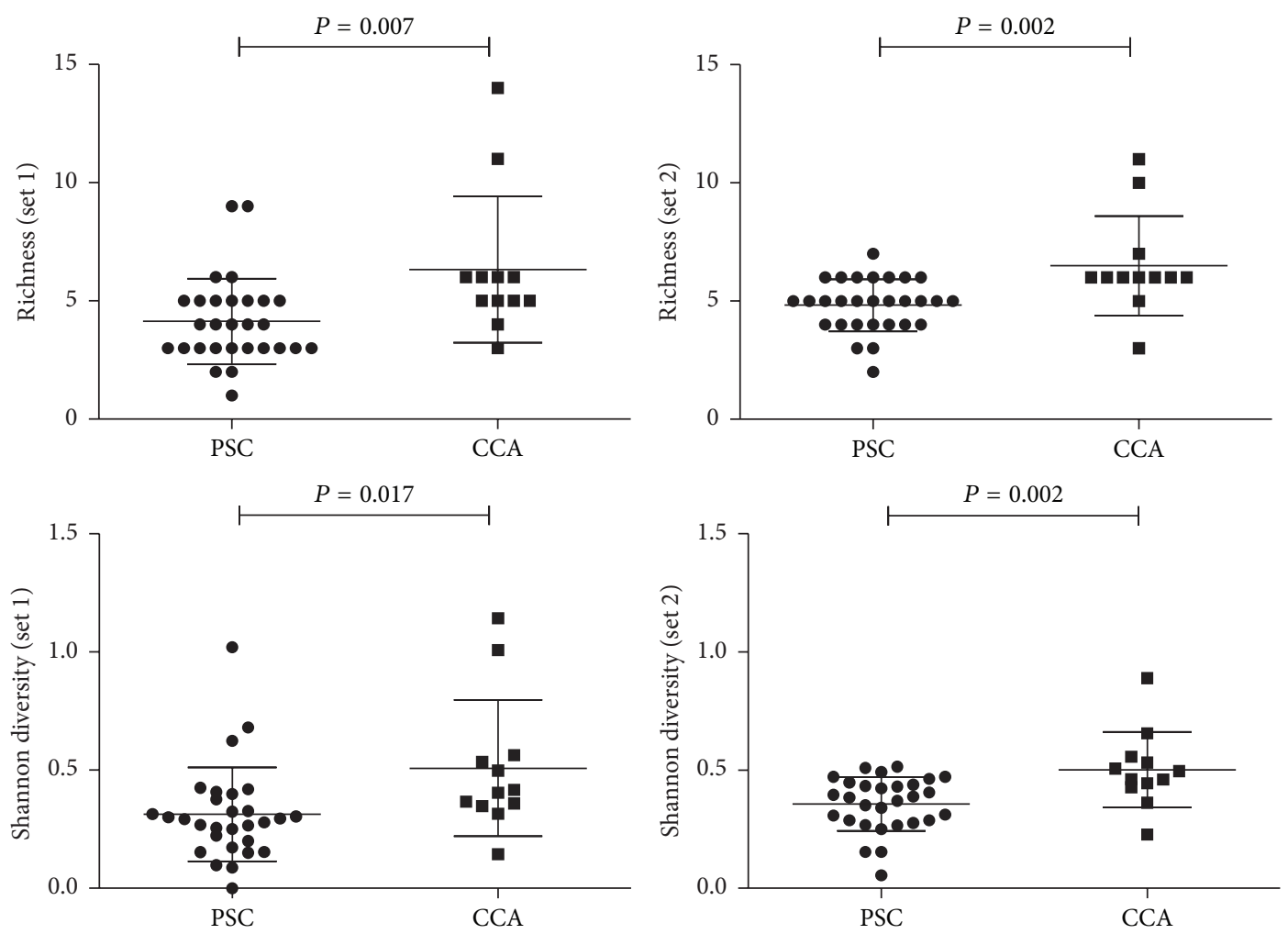

FIgURE 3: Genetic clonal diversity in primary sclerosing cholangitis and cholangiocarcinoma. $P$ values are compared with use of Student's $t$-test.

studies on premalignant diseases such as Barrett's esophagus where an increase in genetic diversity is observed as patients approach the diagnosis of esophageal adenocarcinoma [21, 31]. Interestingly, these findings were independent of the probe set used for the diversity analysis. The best performing measures of clonal diversity were richness (set 2) and the Shannon index (set 1). They had an AUC that was similar to the diagnostic accuracy of the combination of cytology and $M Y C$. The use of cytology combined with diversity measures did not yield substantial improvement and the AUC only slightly increased from 0.78 to 0.80 for both measures.

The diagnostic value of $M Y C$ abnormalities is in accordance with the findings of a recent study that evaluated a novel pancreatobiliary FISH probe set in a large cohort and compared it to the UroVysion probe set [32]. The probes in the panel (1q21, 7p12, 8q24, and 9p21) were first evaluated on 30 selected samples of tumor tissue (CCA and pancreatic cancer) where gain of 8q24 (MYC) was observed in $44 \%$ of the tumor cells analyzed. Subsequently, the authors performed a retrospective analysis of brush samples from 272 patients who underwent ERCP for evaluation of malignancy and found that the presence of polysomy ( $\geq 5$ cells in a sample with copy number gain of at least 2 of the 4 probes), assayed by the new probe set, resulted in an improved sensitivity of $65 \%$ when compared to the UroVysion probe set (sensitivity 46\%) for the detection of pancreatobiliary malignancies. Because the 8 q24 probe was only evaluated in combination with the other probes to diagnose polysomy, the diagnostic value of $8 \mathrm{q} 24$ as a single marker remained unclear.
In our study, although the number of cases was small, MYC had a sensitivity of $100 \%$ and a specificity of $79 \%$ for the diagnosis of CCA in PSC. The MYC (or c-myc) protooncogene is a regulator gene that encodes for a transcription factor and plays a central role in regulating cell cycle proliferation, neoplastic transformation, and apoptosis and MYC abnormalities have been found in many cancers including CCA [33]. In a mouse model of chronic cholestasis, developed to study the mechanisms by which cholestasis contributes to biliary carcinogenesis, MYC was upregulated during CCA progression and resulted in induction of cyclin D1, a protein that contributes to dedifferentiation and cell proliferation in CCA [34]. In addition, knockdown of $M Y C$ reduced progression to CCA. MYC abnormalities have also been described in an inflammatory setting. Komori et al. showed that stimulation by the proinflammatory cytokine tumor necrosis factor-alpha was associated with aberrant expression of the mutagenic enzyme activation-induced cytidine deaminase (AID) in human cholangiocarcinomaderived cells resulting in somatic mutations of several genes including TP53, KRAS, and MYC [17]. Interestingly, MYC abnormalities were not only more present in malignant (PSC) strictures compared to benign strictures, but also more frequent in PSC-CCA than in sporadic CCA (100\% versus $44 \%)$. Although this difference was not statistically significant (most likely due to small numbers), this may indicate that the genetic profile of CCAs arising in PSC differs from sporadic CCA due to the inflammatory setting that may play a more important role in the PSC-associated CCAs. 
Our findings of CEP17 abnormalities being associated with CCA are in line with previous studies that evaluated copy number alterations of chromosomes 3,7 , and 17 to evaluate the presence of polysomy which yields a sensitivity and specificity of approximately $51 \%$ and $93 \%$, respectively. In contrast to our findings, previous studies have reported abnormalities of TP53 as frequent events in CCA [35]. In our analysis, losses of TP53 were not seen at high levels and were not significantly different between PSC and PSC-associated CCA. Part of the discrepancy may be due to differences in detection techniques. Abnormalities of TP53 are usually point mutations, which cannot be detected by FISH, and our study may have underestimated TP53 abnormalities. In addition, most of these studies were performed on resection specimens and may represent more advanced disease.

A major limitation of our study should be considered. Only twelve patients in our study had a confirmed CCA of which three had underlying PSC. Nevertheless, we found significant differences in genetic abnormalities between patients with malignant and benign strictures in PSC. The results of our study suggest a potential role for evaluating genetic markers on brushing specimens, which should include the evaluation of MYC abnormalities and measures of clonal diversity, and further studies with larger cohorts are needed to confirm our findings.

\author{
Abbreviations \\ CCA: Cholangiocarcinoma \\ PSC: Primary sclerosing cholangitis \\ FISH: Fluorescence in situ hybridization \\ AUC: Area under the curve \\ ERCP: Endoscopic retrograde cholangiopancreatography \\ IQR: Interquartile range \\ CI: Confidence interval.
}

\section{Competing Interests}

Kausilia K. Krishnadath reports nonfinancial support from Abbott Molecular during the conduct of the study. In addition, Kausilia K. Krishnadath has a patent issued on the probe set described in this paper.

\section{Acknowledgments}

This study was partly funded by an unrestricted grant from the Ronald Ober Legacy.

\section{References}

[1] S. A. Abrendt, H. A. Pitt, A. Nakeeb et al., "Diagnosis and management of cholangiocarcinoma in primary sclerosing cholangitis," Journal of Gastrointestinal Surgery, vol. 3, no. 4, pp. 357-368, 1999.

[2] K. Boonstra, R. K. Weersma, K. J. van Erpecum et al., "Population-based epidemiology, malignancy risk, and outcome of primary sclerosing cholangitis," Hepatology, vol. 58, no. 6, pp. 2045-2055, 2013.
[3] M. H. Chapman, G. J. M. Webster, S. Bannoo, G. J. Johnson, J. Wittmann, and S. P. Pereira, "Cholangiocarcinoma and dominant strictures in patients with primary sclerosing cholangitis: a 25-year single-centre experience," European Journal of Gastroenterology and Hepatology, vol. 24, no. 9, pp. 1051-1058, 2012.

[4] E. Sinakos, A. K. Saenger, J. Keach, W. R. Kim, and K. D. Lindor, "Many patients with primary sclerosing cholangitis and increased serum levels of carbohydrate antigen 19-9 do not have cholangiocarcinoma," Clinical Gastroenterology and Hepatology, vol. 9, no. 5, pp. 434.el-439.el, 2011.

[5] L. Halme, J. Arola, K. Numminen, L. Krogerus, H. Mäkisalo, and M. Färkkilä, "Biliary dysplasia in patients with primary sclerosing cholangitis: additional value of DNA ploidity," Liver International, vol. 32, no. 5, pp. 783-789, 2012.

[6] P. G. K. Venkatesh, U. Navaneethan, B. Shen, and A. J. McCullough, "Increased serum levels of carbohydrate antigen 19-9 and outcomes in primary sclerosing cholangitis patients without cholangiocarcinoma," Digestive Diseases and Sciences, vol. 58, no. 3, pp. 850-857, 2013.

[7] C. Y. Ponsioen, S. M. E. Vrouenraets, A. W. M. van Milligen de Wit et al., "Value of brush cytology for dominant strictures in primary sclerosing cholangitis," Endoscopy, vol. 31, no. 4, pp. 305-309, 1999.

[8] P. S. Furmanczyk, V. S. Grieco, and S. N. Agoff, "Biliary brush cytology and the detection of cholangiocarcinoma in primary sclerosing cholangitis: evaluation of specific cytomorphologic features and CA19-9 levels," American Journal of Clinical Pathology, vol. 124, no. 3, pp. 355-360, 2005.

[9] L. E. Moreno Luna, B. Kipp, K. C. Halling et al., "Advanced cytologic techniques for the detection of malignant pancreatobiliary strictures," Gastroenterology, vol. 131, no. 4, pp. 1064-1072, 2006.

[10] P. Charatcharoenwitthaya, F. B. Enders, K. C. Halling, and K. D. Lindor, "Utility of serum tumor markers, imaging, and biliary cytology for detecting cholangiocarcinoma in primary sclerosing cholangitis," Hepatology, vol. 48, no. 4, pp. 1106-1117, 2008.

[11] A. Bergquist, B. Tribukait, H. Glaumann, and U. Broomé, "Can DNA cytometry be used for evaluation of malignancy and premalignancy in bile duct strictures in primary sclerosing cholangitis?" Journal of Hepatology, vol. 33, no. 6, pp. 873-877, 2000.

[12] S. C. McKay, K. Unger, S. Pericleous et al., "Array comparative genomic hybridization identifies novel potential therapeutic targets in cholangiocarcinoma," $H P B$, vol. 13, no. 5, pp. 309-319, 2011.

[13] J. Wang, X. Wang, S. Xie et al., "P53 status and its prognostic role in extrahepatic bile duct cancer: a meta-analysis of published studies," Digestive Diseases and Sciences, vol. 56, no. 3, pp. 655662, 2011.

[14] M. R. Timmer, U. Beuers, P. Fockens et al., "Genetic and epigenetic abnormalities in primary sclerosing cholangitisassociated cholangiocarcinoma," Inflammatory Bowel Diseases, vol. 19, no. 8, pp. 1789-1797, 2013.

[15] M. Jaiswal, N. F. LaRusso, and G. J. Gores, "Nitric oxide in gastrointestinal epithelial cell carcinogenesis: linking inflammation to oncogenesis," American Journal of PhysiologyGastrointestinal and Liver Physiology, vol. 281, no. 3, pp. G626G634, 2001.

[16] E. P. Berthiaume and J. Wands, "The molecular pathogenesis of cholangiocarcinoma," Seminars in Liver Disease, vol. 24, no. 2, pp. 127-137, 2004. 
[17] J. Komori, H. Marusawa, T. Machimoto et al., "Activationinduced cytidine deaminase links bile duct inflammation to human cholangiocarcinoma," Hepatology, vol. 47, no. 3, pp. 888896, 2008.

[18] U. Navaneethan, B. Njei, P. G. K. Venkatesh, J. J. Vargo, and M. A. Parsi, "Fluorescence in situ hybridization for diagnosis of cholangiocarcinoma in primary sclerosing cholangitis: a systematic review and meta-analysis," Gastrointestinal Endoscopy, vol. 79, no. 6, pp. 943-950.e3, 2014.

[19] T. A. Gonda, M. P. Glick, A. Sethi et al., "Polysomy and p16 deletion by fluorescence in situ hybridization in the diagnosis of indeterminate biliary strictures," Gastrointestinal Endoscopy, vol. 75, no. 1, pp. 74-79, 2012.

[20] L. E. Vasilieva, S. I. Papadhimitriou, A. Alexopoulou et al., "An extended fluorescence in situ hybridization approach for the cytogenetic study of cholangiocarcinoma on endoscopic retrograde cholangiopancreatography brushing cytology preparations," Human Pathology, vol. 44, no. 10, pp. 2173-2179, 2013.

[21] C. C. Maley, P. C. Galipeau, J. C. Finley et al., "Genetic clonal diversity predicts progression to esophageal adenocarcinoma," Nature Genetics, vol. 38, no. 4, pp. 468-473, 2006.

[22] S. A. Ahrendt, C. F. Eisenberger, L. Yip et al., "Chromosome 9p21 loss and p16 inactivation in primary sclerosing cholangitisassociated cholangiocarcinoma," Journal of Surgical Research, vol. 84, no. 1, pp. 88-93, 1999.

[23] K. Shiraishi, K. Okita, N. Kusano et al., "A comparison of DNA copy number changes detected by comparative genomic hybridization in malignancies of the liver, biliary tract and pancreas," Oncology, vol. 60, no. 2, pp. 151-161, 2001.

[24] M. Taniai, H. Higuchi, L. J. Burgart, and G. J. Gores, "p16 INK4a promoter mutations are frequent in primary sclerosing cholangitis (PSC) and PSC-associated cholangiocarcinoma," Gastroenterology, vol. 123, no. 4, pp. 1090-1098, 2002.

[25] Y. Ukita, M. Kato, and T. Terada, "Gene amplification and mRNA and protein overexpression of c-erbB-2 (HER-2/neu) in human intrahepatic cholangiocarcinoma as detected by fluorescence in situ hybridization, in situ hybridization, and immunohistochemistry," Journal of Hepatology, vol. 36, no. 6, pp. 780-785, 2002.

[26] J.-Y. Lee, Y.-N. Park, K.-O. Uhm, S.-Y. Park, and S.-H. Park, "Genetic alterations in intrahepatic cholangiocarcinoma as revealed by degenerate oligonucleotide primed PCR-comparative genomic hybridization," Journal of Korean Medical Science, vol. 19, no. 5, pp. 682-687, 2004.

[27] K. Nakazawa, Y. Dobashi, S. Suzuki, H. Fujii, Y. Takeda, and A. Ooi, "Amplification and overexpression of c-erbB-2, epidermal growth factor receptor, and c-met in biliary tract cancers," The Journal of Pathology, vol. 206, no. 3, pp. 356-365, 2005.

[28] M. B. Cohen, R. J. Wittchow, F. C. Johlin, K. Bottles, and S. S. Raab, "Brush cytology of the extrahepatic biliary tract: comparison of cytologic features of adenocarcinoma and benign biliary strictures," Modern Pathology, vol. 8, no. 5, pp. 498-502, 1995.

[29] A. A. Renshaw, R. Madge, M. Jiroutek, and S. R. Granter, "Bile duct brushing cytology: statistical analysis of proposed diagnostic criteria," American Journal of Clinical Pathology, vol. 110, no. 5, pp. 635-640, 1998.

[30] A. M. Rygiel, J. W. P. M. Van Baal, F. Milano et al., "Efficient automated assessment of genetic abnormalities detected by fluorescence in situ hybridization on brush cytology in a Barrett esophagus surveillance population," Cancer, vol. 109, no. 10, pp. 1980-1988, 2007.
[31] X. Li, P. C. Galipeau, T. G. Paulson et al., "Temporal and spatial evolution of somatic chromosomal alterations: a case-cohort study of Barrett's esophagus," Cancer Prevention Research, vol. 7, no. 1, pp. 114-127, 2014.

[32] E. G. Barr Fritcher, J. S. Voss, S. M. Brankley et al., "An optimized set of fluorescence in situ hybridization probes for detection of pancreatobiliary tract cancer in cytology brush samples," Gastroenterology, vol. 149, no. 7, pp. 1813.el-1824.el, 2015.

[33] L. Soucek and G. I. Evan, "The ups and downs of Myc biology," Current Opinion in Genetics and Development, vol. 20, no. 1, pp. 91-95, 2010.

[34] H. Yang, T. W. H. Li, J. Peng et al., "A mouse model of cholestasis-associated cholangiocarcinoma and transcription factors involved in progression," Gastroenterology, vol. 141, no. 1, pp. 378.e4-388.e4, 2011.

[35] S. A. Ahrendt, A. Rashid, J. T. Chow, C. F. Eisenberger, H. A. Pitt, and D. Sidransky, "p53 overexpression and K-ras gene mutations in primary sclerosing cholangitis-associated biliary tract cancer," Journal of Hepato-Biliary-Pancreatic Surgery, vol. 7, no. 4, pp. 426-431, 2000 


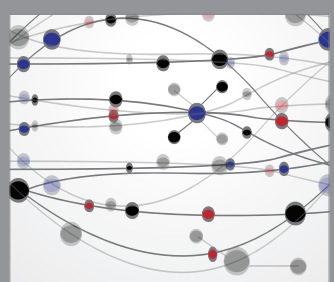

The Scientific World Journal
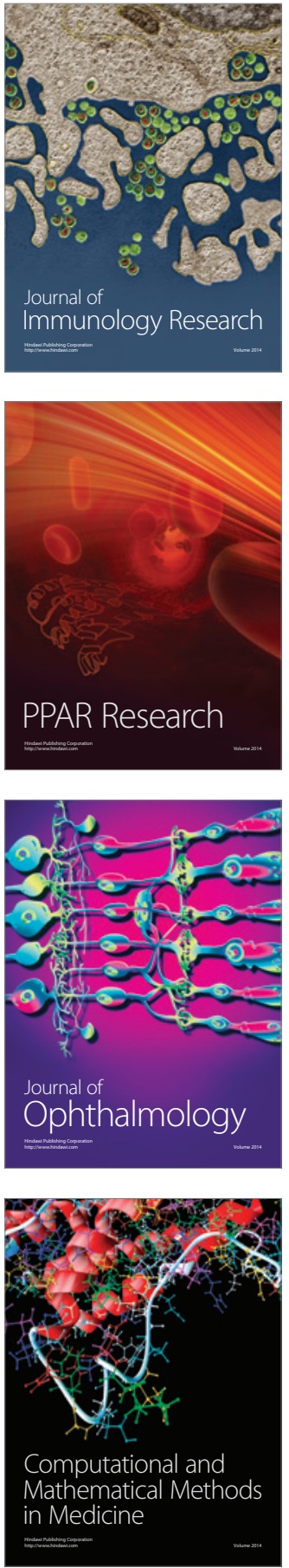

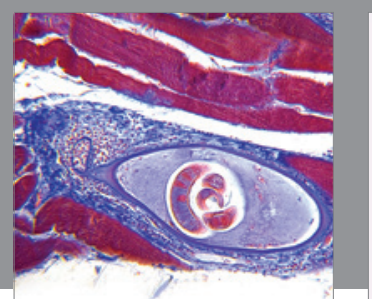

Gastroenterology Research and Practice

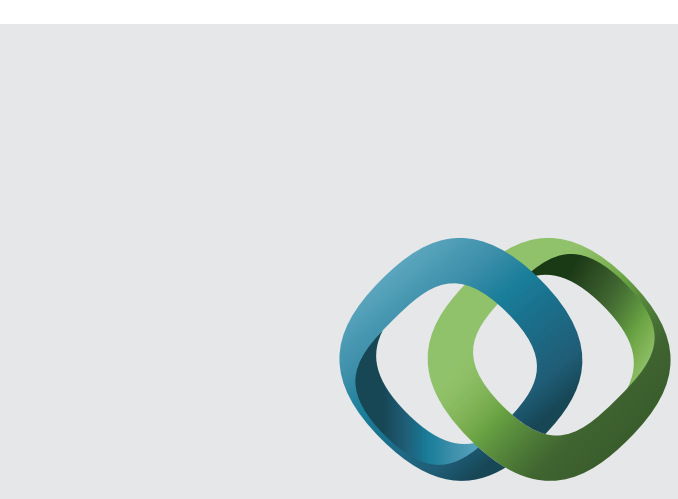

\section{Hindawi}

Submit your manuscripts at

http://www.hindawi.com
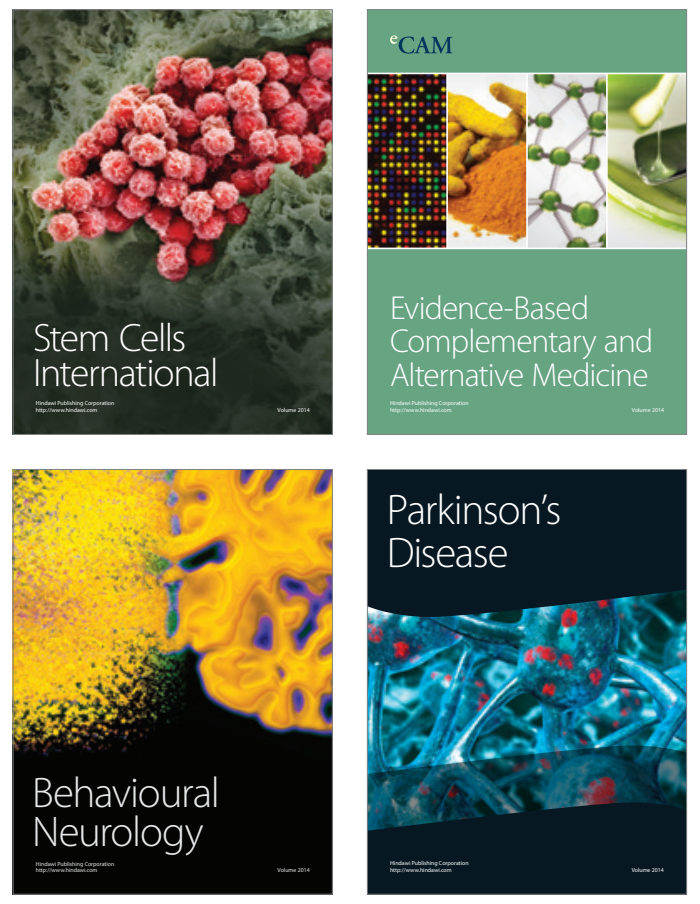
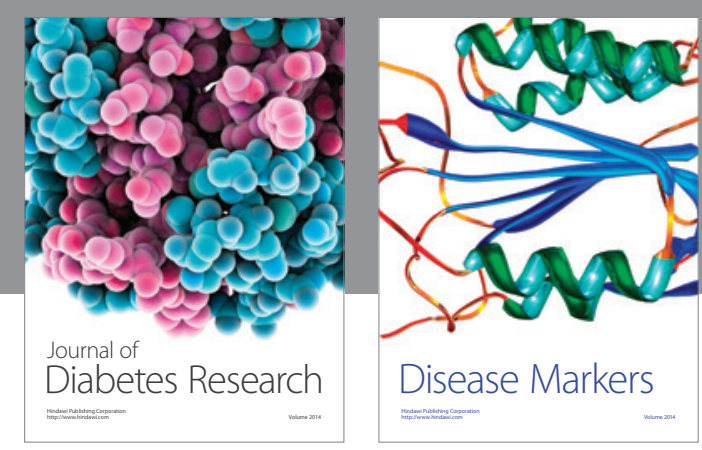

Disease Markers
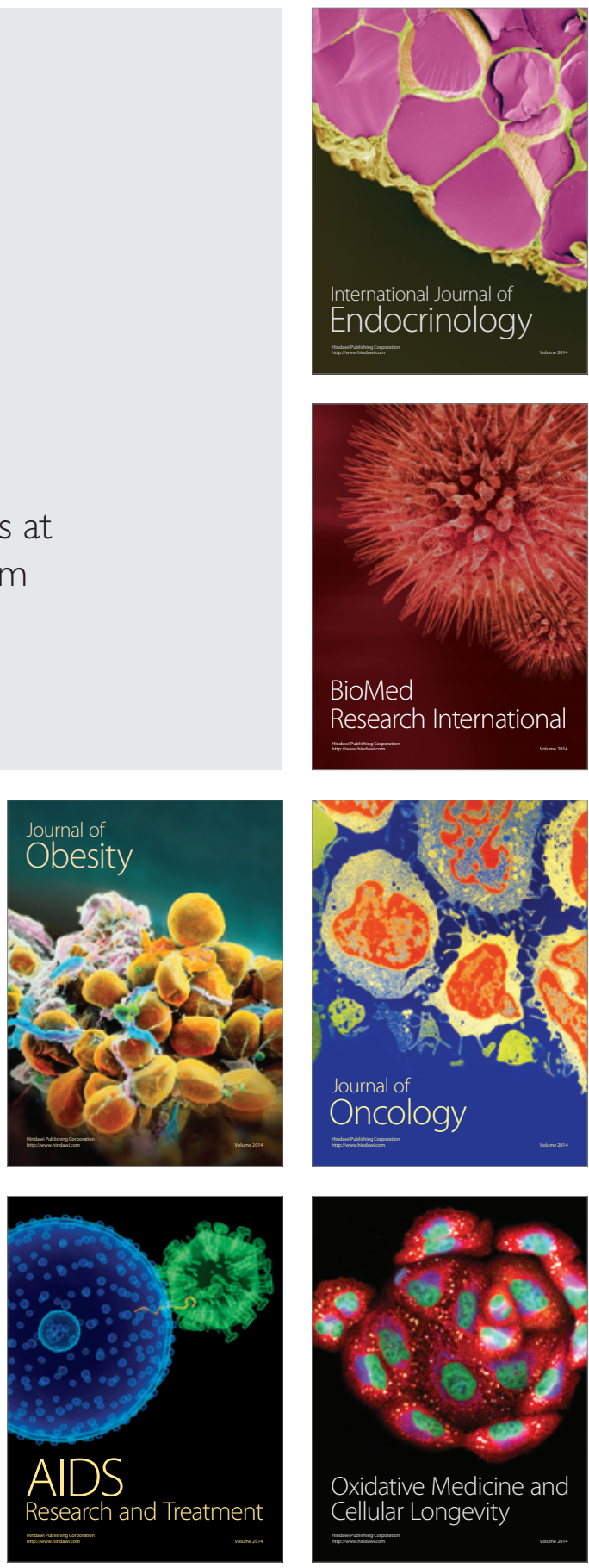